\title{
Forget About Cleaning up Your Micrographs: Deep Learning Segmentation is Robust to Image Artifacts
}

\author{
Peng Dong ${ }^{1}$, Benjamin Provencher ${ }^{2}$, Nabil Basim ${ }^{1}$, Nicolas Piché ${ }^{2}$ and Mike Marsh ${ }^{3}$ \\ ${ }^{1}$ McMaster University, Hamilton, Ontario, Canada, ${ }^{2}$ Object Research Systems, Montreal, Quebec, \\ Canada, ${ }^{3}$ Object Research Systems, Denver, Colorado, United States
}

Quantitative image analysis almost always requires a thorough segmentation, i.e. a pixel-wise labeling of the different phases of the imaged material. With traditional tools, that segmentation can proceed only after complicating image artifacts have been filtered away or otherwise compensated for so they do not lead to spurious labeling. Identifying the right image processing operations to mitigate artifacts and restore images can be tedious, and executing those operations may introduce side-effects in the form of image processing artifacts. We show here that Deep Learning segmentation, image processing by convolutional neural networks, produces high quality labeling without any prior image cleanup, greatly simplifying the workflow for yielding valuable quantitative analyses.

We performed serial-section imaging to collect a 3D image stack of an ordinary Portland cement sample mortar sample with water/cement ratio of 0.485 sample, using an FEI Helios nanoLab PFIB-SEM dualbeam system(ThermoFisher, Inc) at the Canadian Centre for Electron Microscopy, housed at McMaster University. The full stack was 156 slices, where each slice has a horizontal field-of-view of 96.7 microns with image dimensions of 3868 x 4062 , giving us a total of 2450 Mpixel of image data for segmentation. As customary in PFIB, the Xe milling introduced strong curtaining artifacts that manifest as linear grooves at varying angles across the imaged surface of the sample. For each imaged slice, we acquired both in chamber electron detector (ICE) and through-the-lens detector (TLD) signals with an e-beam landing voltage of $2 \mathrm{keV}$. Also, common in these experiments, the evolving sample geometry introduced occlusion between the imaged surface and the detector; this manifests in non-uniform detector response across the imaged plane. That means that the grayscale intensity for the same phase will vary across the image, further complicating proper image segmentation. Lastly, under these imaging conditions, both image channels, the ICE in particular, are further frustrated by high noise levels.

Previous work has shown that convolutional neural networks--initially with the U-Net architecture [1]--, are powerful tools for fully automated image segmentation. Here, we evaluate two architectures, U-Net and Sensor-3D [2], but we vary the input signals and the number of input slices. In total, we trained and tested nine different models to explore the benefits of simultaneously processing multi-slice, multichannel image data. All models were trained with a selection of three unprocessed slices, totaling 36 Mpixel, paired with the manual segmentations of the same slices. This manual segmentation served as the ground truth for the target segmentation and was the target result that the network models were learning to reproduce. From that training dataset, $25 \%$ (9.2MPixel) of the data were reserved for validation. An independent fourth slice was used for evaluation. We performed the manual segmentation, as well as configuration, training, and evaluation of the neural network models with Dragonfly (Object Research Systems, Inc.) [3].

We find that the model's behavior benefits from both experimental variables, i.e. Deep Learning image segmentation accuracy improves when including both signals and when the models consider the signal from the preceding and subseuqnet slices. Importantly, we find that the results of the best models demonstrate that fully automated image segmentation by Deep Learning yields high-confidence, 
quantification-ready results without having to pre-process the images to clean up the noise or the curtaining and non-uniform response artifacts.

The successful segmentation of research samples without having to work out image cleanup procedures is certain to increase the throughput of non-routine research image analysis. We expect this capability of Deep Learning will transcend other common imaging artifacts not encountered here. As such, Deep Learning stands out as a robust solution, emerging to become the standard for research microscopy applications.

\section{References}

[1] O. Ronnenberger, et al., Medical Image Computing and Computer-Assisted Intervention 9351 (2015) p. 234-241.

[2] A.A. Nivikov, et al., IEEE Trans Med Imaging 38 (2019), p. 1207-1215.

[3] R. Makovetsky, et al., Microscopy and Microanalysis 24 (Suppl 1) (2018), p. 532-533. 